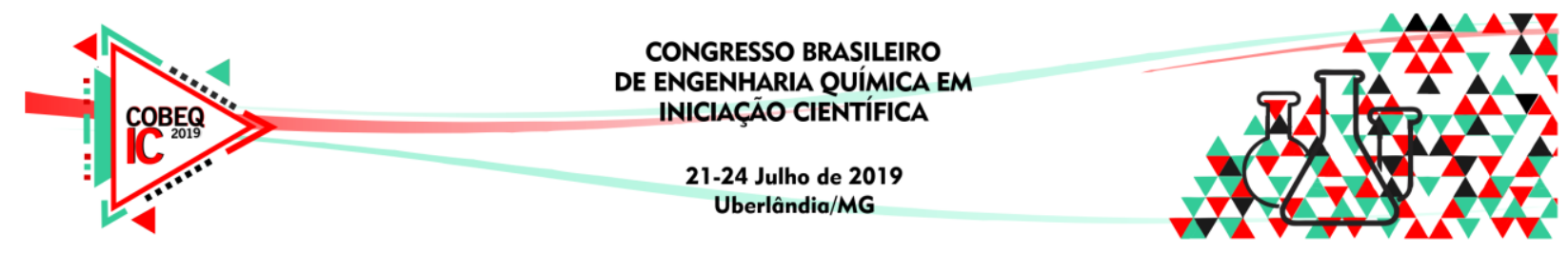

\title{
ESTUDO COMPARATIVO DA OBTENÇÃO DE BIODIESEL A PARTIR DE ÓLEO DE MAMONA EM LABORATÓRIO E NO SIMULADOR DE PROCESSOS QUÍMICOS DWSIM
}

\author{
A. L. A. BAPTISTA ${ }^{1}$, C. C. AMARAL ${ }^{2}$ e M.V. RIBEIRO ${ }^{3}$ \\ ${ }^{1,2,3}$ Centro Universitário Newton Paiva, Faculdade de Ciências Exatas e Tecnológicas \\ E-mail: annaluizaaraujobaptista@gmail.com
}

\begin{abstract}
RESUMO - A produção de biodiesel e o uso de simuladores de processos químicos são temas atuais na Engenharia Química. Neste artigo o software DWSIM foi utilizado para modelar a obtenção de biodiesel a partir do óleo de mamona. A síntese em laboratório foi realizada através da reação de transesterificação do óleo de rícino com metanol em meio básico. Os produtos da reação são o biodiesel e a glicerina que foram caracterizados por espectroscopia na região do infravermelho por transformada de Fourier (FTIR). As condições da síntese em laboratório foram utilizadas para confeccionar o modelo virtual no DWSIM. As análises estatísticas indicaram boa compatibilidade entre os resultados alcançados na síntese em laboratório e no modelo virtual. O DWSIM é uma ferramenta interessante no estudo de variáveis que possibilitem a otimização de processos industriais.
\end{abstract}

\section{INTRODUÇÃO}

\subsection{BIODIESEL}

Pela definição da lei 11.097 de 13/01/2005, o biodiesel pode ser classificado como um combustível alternativo, de natureza renovável, que possa oferecer vantagens socioambientais ao ser empregado na substituição total ou parcial do diesel de petróleo em motores de ignição por compressão interna (Ciclo Diesel). Pode ser produzido a partir de gorduras animais ou de óleos vegetais, existindo dezenas de espécies vegetais no Brasil que podem ser utilizadas para obtenção de biodiesel com a mamona, dendê (palma), girassol, babaçu, amendoim, pinhão manso e soja, dentre outras.

\subsection{MAMONA}

A mamona (Ricinus Communis L.) é uma planta de origem afro-asiática que pode ser encontrada em todo o Brasil em virtude de ser facilmente adaptável a diversas condições de solo e clima. Ela é uma planta euforbiácea, sendo sua semente tóxica devido a uma proteína chamada ricina. O principal produto da mamona é o óleo, também chamado óleo de rícino. É uma importante matéria-prima para a indústria química, sendo utilizado na composição de inúmeros produtos como tintas, vernizes, cosméticos, lubrificantes, plásticos etc. A lista de produtos feitos a partir do óleo de mamona é abrange mais de 400 itens. Esse óleo possui 


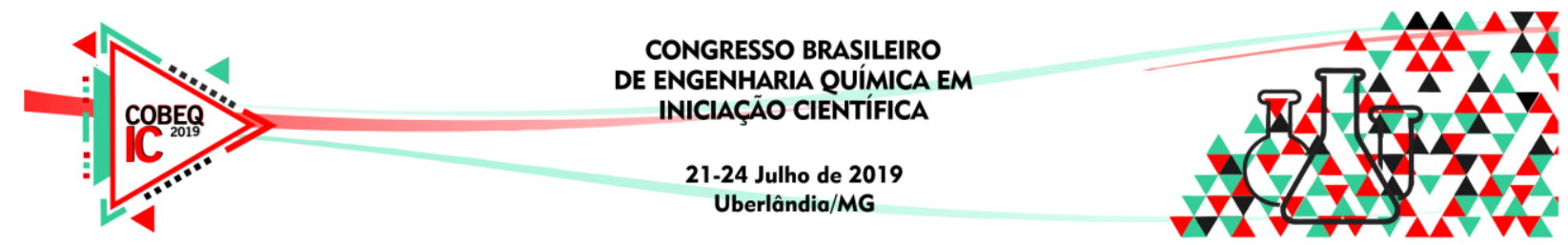

características químicas que lhe tornam único na natureza: é composto exclusivamente (90\%) por um único ácido graxo (ácido ricinoleico) que contém uma hidroxila que lhe torna solúvel em álcool a baixa temperatura, muito viscoso e com propriedades físicas especiais (SCHNEIDER, 2002).

$\mathrm{Na}$ indústria, a extração do óleo de rícino pode ser feita da semente completa ou da baga (semente mecanicamente descascada). O método usado para extrair o óleo pode ser prensagem, a frio ou a quente, ou extração por solvente. A semente primeiramente é limpa e depois é direcionada para o aquecimento de diversos estágios, em uma chaleira. É prensado e degomado. A degomagem é realizada com água ou vapor de água, elevando a umidade do extrato a $45 \%$. Com isso, o óleo aumenta o volume e é imediatamente filtrado ou passa por uma centrífuga de volume (Super decanter). A borra obtida na degomagem é misturada à torta que vai à extração por solvente, de onde se obtêm o óleo final e o farelo. O óleo obtido da prensagem, então, é clarificado com adsorvente e seco. Nesta operação é misturado com o óleo proveniente da extração por solvente. Todas as terras usadas na clarificação com cerca de $30 \%$ de óleo seguem com a torta para extração por solvente (SCHNEIDER, 2002).

\subsection{MODELAGEM COMPUTACIONAL}

A modelagem computacional utiliza um conjunto de métodos, ferramentas e formulações direcionadas à solução de problemas complexos, envolvendo grande número de variáveis, volumosa massa de dados, processamento e manipulação de imagens. Ela permite que se criem modelos computacionais para situações em que é impossível ou muito caro testar ou medir as diversas soluções possíveis para um fenômeno a partir de modelos experimentais ou por soluções analíticas.

O simulador de processos químicos DWSIM é um ambiente aberto, brasileiro, que segue o padrão CAPE-OPEN e está disponível para ser utilizado em Windows, Linux e Mac. O DWSIM permite que estudantes e engenheiros químicos estudem e compreendam comportamentos em processos químicos usando modelos rigorosos em operações termodinâmicas instaladas no software como misturador, separador, bomba, compressor, expansor, aquecedor, refrigerador, válvula, colunas de destilação, trocador de calor, vários reatores, separadores de sólidos, etc.

Alguns pacotes de propriedades mais populares disponíveis no software são PengRobinson, Chao-Seader, Grayson-Streed, gás ideal, UNIFAC, Soave-Redlich-Kwong, LeeKesler e outras. Ele possui biblioteca de componentes para pesquisa instantânea; ferramenta de geração de gráficos e curvas; detecção automática de erros; disponibilidade para uso em dispositivos móveis; interface simples para usuários com pouco conhecimento de programação; gerenciamento dos fluxos de materiais, reações químicas e criação de componentes; projetar uma representação esquemática do processo, etc.

O presente trabalho estudou a viabilidade do uso do simulador DWSIM na modelagem do processo de transesterificação do óleo de mamona para obtenção de biodiesel em comparação com os resultados da síntese em laboratório. 


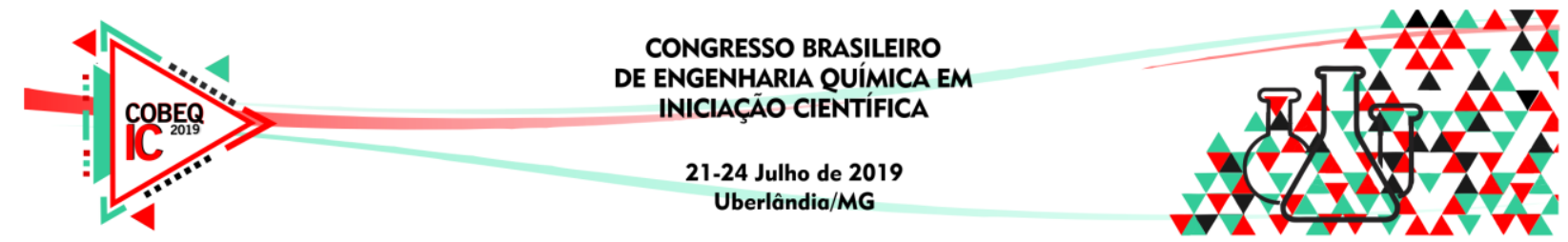

\section{MATERIAIS E MÉTODOS}

\subsection{Síntese do Biodiesel}

No laboratório mediu-se $25 \mathrm{~mL}$ de óleo de mamona em uma proveta que foi transferido para um béquer de $250 \mathrm{~mL}$ para que fosse aquecido em uma chapa aquecedora. Ao mesmo tempo em outro béquer dissolveu-se $0,1968 \mathrm{~g}$ de $\mathrm{NaOH}$ em $6,3 \mathrm{ml}$ de metanol. Após o aquecimento do óleo, adicionou-se então a mistura de metanol com $\mathrm{NaOH}$ sob agitação constante. Após a formação da glicerina, a mistura foi transferida para uma proveta, para separação das fases. Após a separação do biodiesel da glicerina, foi realizado a caracterização através da espectroscopia na região do infravermelho (Spectrum Two PerkinElmer FTIR Spectrometer). A síntese foi realizada nas temperaturas de $61,70,92$ e $102^{\circ} \mathrm{C}$.

\subsection{Modelagem e Simulação}

Os dados dos experimentos em laboratório foram coletados e plotados no gráfico de rendimento versus temperatura para entender o comportamento da reação de transesterificação do óleo de mamona. A equação da reta obtida com os dados anteriores foi inserida no DWSIM. O modelo virtual do processo foi confeccionado, testado e validado no software. Com os resultados obtidos na síntese em laboratório e no modelo virtual no simulador realizou-se testes comparativos que foram validados por análises estatísticas.

\section{RESULTADOS E DISCUSSÕES:}

\subsection{Síntese de Biodiesel}

A reação de transesterificação permitiu obter o biodiesel de rícino e a glicerina evidenciado por uma mudança de coloração e redução de viscosidade do biodiesel em relação ao óleo de rícino, conforme mostrado na figura 1:

Figura 1 - Biodiesel.

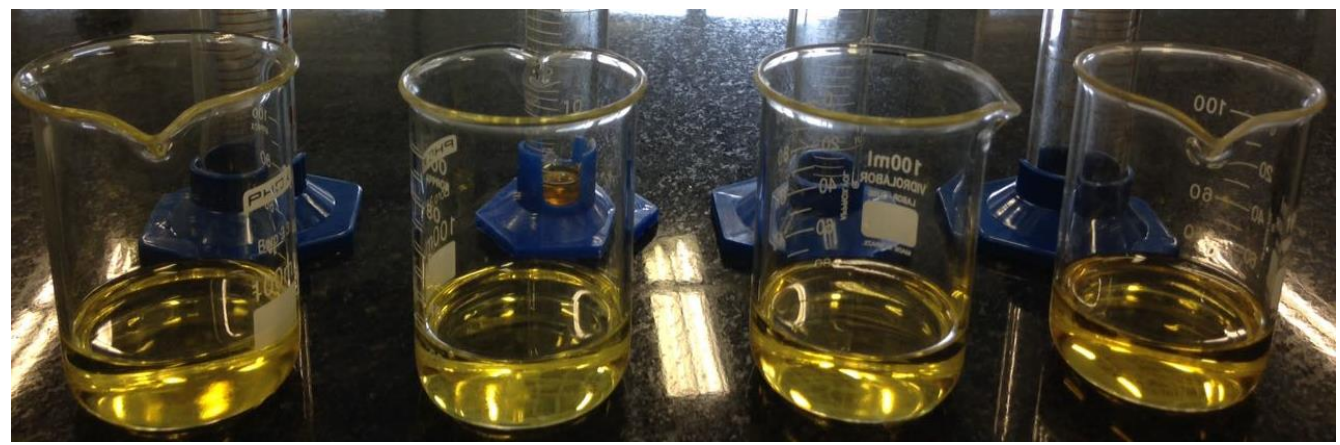

Os produtos da síntese do biodiesel através da transesterificação do óleo de rícino foram caracterizados por espectroscopia na região do infravermelho (FTIR) e os dados foram agrupados na tabela 1: 


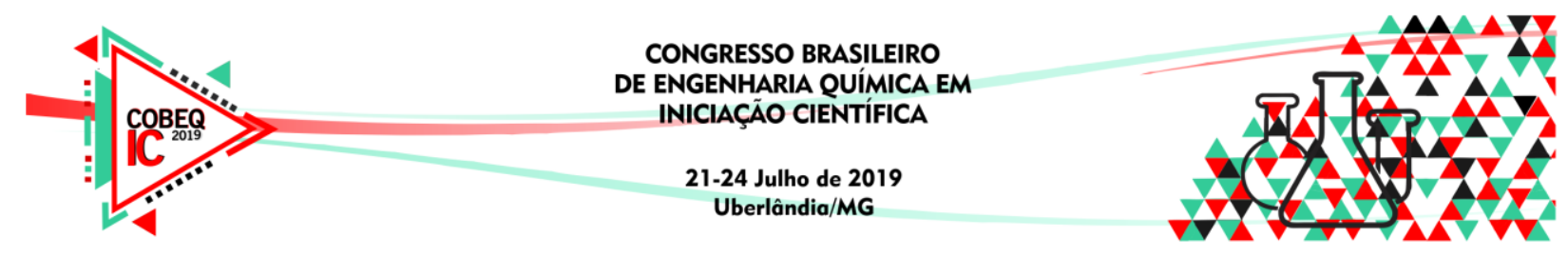

Tabela 1 - Identificação das bandas de deformação axial do óleo de mamona, glicerina e biodiesel.

\begin{tabular}{cccc}
\hline Deformação Axial & \multicolumn{3}{c}{ Faixa de Transmitância $\left(\mathbf{c m}^{-1}\right)$} \\
& Óleo de Rícino & Glicerina & Biodiesel \\
\hline C-OH & 3380,4 & 3292,53 & 3350,97 \\
C-H & 2924,34 & 2930,55 & 2925,11 \\
C=O & 1742,85 & - & 1740,68 \\
C=C & - & 1415,7 & - \\
C-C & - & - & 1436,1 \\
C-O & 1458,07 & 1030,89 & 1171,95 \\
\hline
\end{tabular}

Fonte: Autores (2017)

A análise dos espectros de FTIR indicaram a formação do biodiesel na síntese realizada em laboratório através das bandas referentes a deformação axial do grupo carbonila $(\mathrm{C}=\mathrm{O})$ e o estreitamento da deformação axial da ligação $(\mathrm{C}-\mathrm{O})$ que são bandas características dos ésteres.

\subsection{Modelagem e Simulação no DWSIM}

No software DWSIM é possível a inserção de funções matemáticas para melhorar a aderência da simulação à realidade. Sendo assim, a produção simulada do biodiesel traz a exigências para a confecção do modelo matemático representativo a partir dos resultados obtidos em laboratório, vide a Tabela 2:

Tabela 2 - Rendimentos da síntese em laboratório.

\begin{tabular}{ccc}
\hline Amostra & Temperatura $\left({ }^{\mathbf{O}} \mathbf{C}\right)$ & Rendimento (\%) \\
\hline 1 & 334,15 & 94,81 \\
2 & 343,15 & 96,43 \\
3 & 365,15 & 99,26 \\
4 & 375,15 & 100,00 \\
\hline
\end{tabular}

Fonte: Autores (2017)

A partir dos dados da tabela 2 foi possível avaliar o comportamento da reação de transesterificação. As variáveis escolhidas foram a temperatura $(\mathrm{K})$ no eixo x e o rendimento (\%) no eixo y, os dados apresentaram a equação $\mathrm{y}=01286 \mathrm{x}+52,687$ com correlação linear $\mathrm{R}^{2}=0,9857$. Os dados foram submetidos ao teste estatístico de Anderson-Darling onde o pvalor foi 0,488 , sabendo que este valor é maior que 0,10 , os dados seguem uma distribuição normal e comprovou que o experimento possui comportamento linear. 


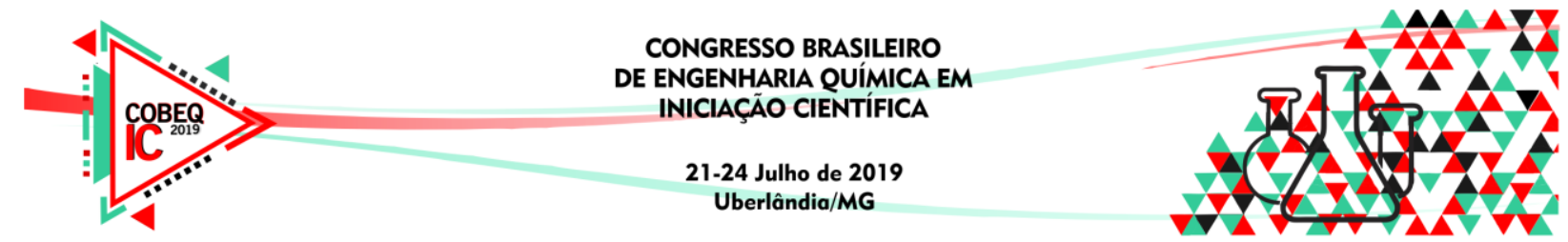

Para a simulação computacional utilizou-se o software DWSIM versão 4.0 de código livre. Para os cálculos termofísicos dos componentes adotou-se o pacote de propriedades UNIQUAC e como plano de execução Nested Loops. Embora exista uma biblioteca vasta no software, as substâncias ácido ricinoléico e ricinoleato de etila não estavam implantadas e foram inseridas via "New Compound Creator Study". Para o modelo virtual foram empregados os seguintes dados para a mistura inicial, vide Tabela 3:

Tabela 3 - Propriedades referentes à Mistura 1.

\begin{tabular}{cc}
\hline Propriedade & Valor \\
\hline Temperatura & $298,15 \mathrm{~K}$ \\
Pressão & $101325 \mathrm{~Pa}$ \\
Fluxo Mássico & $0,5 \mathrm{~kg} . \mathrm{s}^{-1}$ \\
Fluxo Molar & $8,08188 \mathrm{~mol}_{\mathrm{s}} \mathrm{s}^{-1}$ \\
Fluxo Volumétrico & $0,00047 \mathrm{~m}^{3} . \mathrm{s}^{-1}$ \\
Fração Molar - Metanol & 0,5 \\
Fração Molar - Hidróxido de Sódio & 0,4 \\
Fração Molar - Ácido Ricinoléico & 0,1 \\
\hline
\end{tabular}

Fonte: Autores (2017)

No desenvolvimento do modelo virtual, utilizou-se o reator de conversão (RC-001) para simular a reação sob agitação constante, fluxo de massa continuo e em aquecimento. $\mathrm{O}$ misturado (Mixer 1) foi adicionado para obter o modelo virtual mais próximo do experimento real onde são homogeneizadas 2 correntes de massa, uma contendo $\mathrm{CH}_{3} \mathrm{OH}$ e $\mathrm{NaOH}$ e a outra com ácido ricinoléico. O rendimento calculado no software foi determinado pelo modelo matemático linear alcançado. No modelo virtual construído no DWSIM (Figura 2), os objetos em azul simbolizam o sucesso da simulação sem ocorrência de erros:

Figura 2 - Modelo virtual para reação de transesterificação - DWSIM.

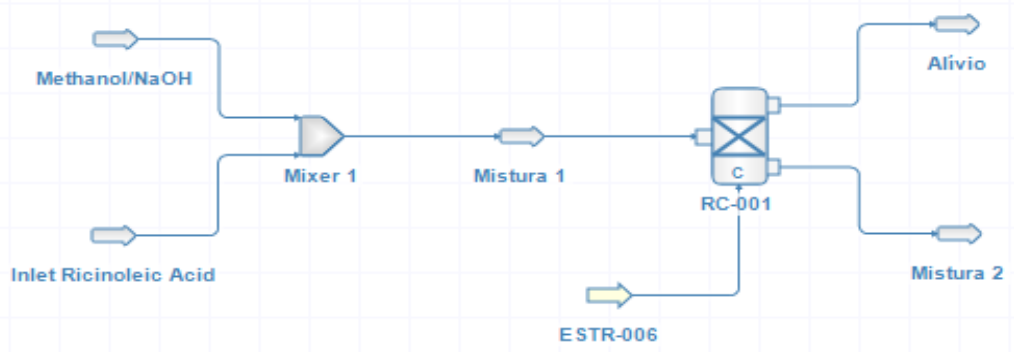




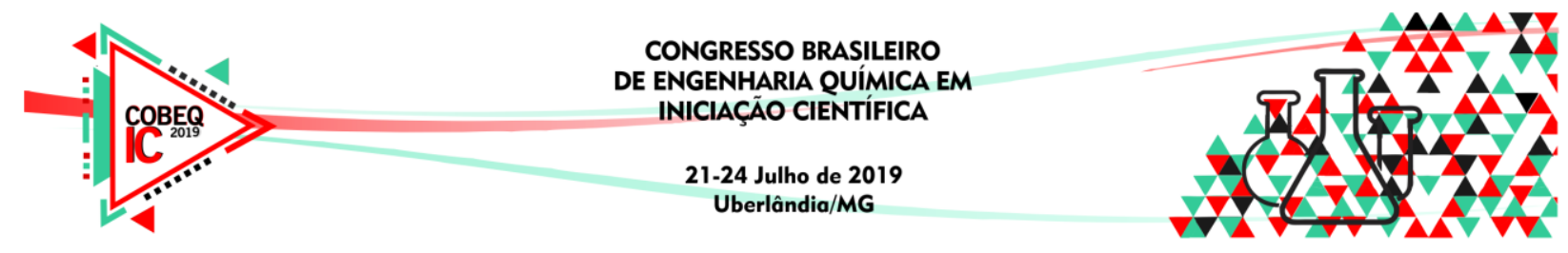

Os resultados obtidos no DWSIM foram comparados com os resultados da síntese e validados pelo método estatístico do teste t pareado com $95 \%$ de confiabilidade. Na tabela 4 temos uma análise comparativa dos rendimentos:

Tabela 4 - Análise comparativa de rendimentos.

\begin{tabular}{cc}
\hline $\begin{array}{c}\text { Rendimento } \\
\text { Síntese }\end{array}$ & $\begin{array}{c}\text { Rendimento } \\
\text { DWSIM }\end{array}$ \\
\hline 0,9455 & 0,9569 \\
0,9606 & 0,9683 \\
0,9273 & 0,9785 \\
0,9606 & 0,9810 \\
1,0000 & 0,9886 \\
1,0000 & 0,9937 \\
\hline \multicolumn{2}{c}{ Fonte: Autores $(2017)$}
\end{tabular}

$O \mathrm{t}_{\text {(experimental) }}$ foi 1,4369 , um valor menor que o $\mathrm{t}_{\text {(tabelado) }}$ que é 2,4469 , isso indica que as variâncias não apresentam diferenças significativas quando comparamos os rendimentos obtidos na síntese e no modelo virtual.

\section{CONCLUSÕES}

Os testes empíricos proporcionaram coletas de dados de temperatura e rendimento da síntese do biodiesel que apresentou comportamento linear e distribuição normal. O DWSIM mostrou-se um simulador de processos químicos eficiente para reproduzir em ambiente virtual os rendimentos alcançados na síntese do biodiesel em laboratório (tabela 4), o que foi validado através de análises estatísticas.

O modelo virtual permitiu realizar teste para determinar as propriedades, fluxo de massa e comportamento dos produtos da reação. O software é uma ferramenta interessante para cálculos de balanço de massa, fluxo, potência de equipamentos e reciclo. A simulação atestou a viabilidade da obtenção do biodiesel a partir do óleo de mamona com um rendimento médio de $97,8 \%$.

\section{REFERÊNCIAS BIBLIOGRÁFICAS}

DWSIM. Home. Disponível em:<http://dwsim.fossee.in/>. Acesso em: 03/03/2017.

KNOTHE, G., KRAHL, J., GERPEN, J.V., RAMOS, L.P. Manual de biodiesel. Rio de Janeiro/RJ: Blucher, 2006, 352p.

SCHENEIDER, R.C.S. Extração, caracterização e transformação do óleo de rícino. Tese (Doutorado em Química). Porto Alegre/RS: Universidade Federal do Rio Grande do Sul, 2002, 240p.

\section{AGRADECIMENTOS}

Ao Centro Universitário Newton Paiva pelo suporte durante a pesquisa. 